\title{
Influence of Spatial Restrictions on Equilibrium Reactions: A Case Study about the Excimer Formation of Pyrene
}

\author{
A. Thomas, ${ }^{\dagger}$ S. Polarz, ${ }^{*, \dagger},+\neq$ and M. Antonietti ${ }^{\dagger}$ \\ Max-Planck Institute of Colloids and Interfaces, Research Campus Golm, 14424 Potsdam, Germany, and \\ Lehrstuhl für Anorganische Chemie I, Ruhr-Universität Bochum, Universitätsstrasse 150, \\ D-44780 Bochum, Germany
}

Received: July 23, 2002; In Final Form: April 1, 2003

\begin{abstract}
(Chemical) processes under spatial confinements are important for a number of areas, as for instance in catalysis (chemistry) or enzymatic reactions (biology). The role of the spatial restriction parallel to the functional interactions in these systems is hard to trace. Ordered mesoporous silica materials are ideal to provide a multiplicity of parallel, just nanometer-large, well-defined confinements. Therefore, they are ideal to act as model compounds for the examination of spatial confinement effects. In the current contribution, a bimolecular reaction, the formation of excimers of pyrene, is studied under varying confinement conditions in ordered mesoporous silica materials. It will be shown that spatial confinement changes both the excimer formation equilibrium and the kinetics of excimer relaxation.
\end{abstract}

\section{Introduction}

Nanochemistry is a new area of chemistry within the broad field of nanotechnology. ${ }^{1-3}$ Nanochemistry is not only one method to produce nanostructures, 4,5 but it also deals "with extraordinary size-dependent effects emerging from confined states of all matter". ${ }^{6}$ A spatial confinement can influence matter differently on different length scales. At the larger nanoscale $(2-50 \mathrm{~nm})$, experiments can be designed for the creation of nanostructures as colloids. At smaller sizes $(1-10 \mathrm{~nm})$, molecular events such as the outcome of a chemical reaction might be influenced by spatial restrictions.

Nanochemistry is preferentially performed in a "reaction flask" of nanosize, just slightly exceeding molecular dimensions. If a given volume could be divided into such small compartments by adiabatic walls, a different situation than that in the continuous $3 \mathrm{D}$ phase is realized. The amount of molecules per confinement, the total energy, and the size and shape of the confinement (volume) are fixed, and diffusional pathways are restricted. In statistical thermodynamics this situation is called the microcanonic ensemble situation, whereas classical conditions chemists are used to, such as a reaction in a solution, represent the canonic ensemble. Reactions under microcanonical or confined conditions are not exotic; they are ubiquitous. Two prominent examples are active centers of enzymes or catalysis in the fine pores of zeolithes. In these cases, the effects of the chemically active site and the spatial restrictions in the confinement cannot be separated from each other. Experimentally, the question how a spatial confinement could influence molecular events, and if there is a transition to the microcanonic ensemble, is rarely treated.

Mesoporous silica materials ${ }^{7-9}$ are the most suitable candidates for the examination of such confinement or nanoreactor effects. $\mathrm{SiO}_{2}$ is stable, chemically inert, and spectroscopically

\footnotetext{
* To whom correspondence should be addressed. E-mail: sebastian. polarz@rub.de.

Max-Planck Institute of Colloids and Interfaces, Research Campus Golm.

+ Ruhr-Universität Bochum.
}

relatively inactive. Pores with well-defined size and shape can be prepared with a variety of template techniques, ${ }^{8,10}$ and with monolith technology, a macroscopic volume $\left(\approx \mathrm{cm}^{3}\right)$ can be effectively divided into nanocompartments $\left(\approx \mathrm{nm}^{3}\right)$ without interstitial grain volume. ${ }^{11-13}$ In general, those confinements are usually employed to confine the growth of nanoparticles, and there are only a few reports where these materials were used for other purposes. ${ }^{14}$ The materials used so far were mostly of the MCM-type with its hexagonally aligned cylindrical pores. ${ }^{15,16}$ Here, monolithic pieces of materials with the so-called "worm-type" pore system were employed which we-due to their shorter pore length-regard as more suitable for confinement examinations (see Figure 1). Additionally, the pore sizes of the materials can be continuously adjusted in the range between 2 and $10 \mathrm{~nm}$ by mixed template routes. ${ }^{17}$

In the current contribution, the question is addressed how the dynamic equilibrium of a binary model reaction is affected by the spatial nanoconfinement within an ordered mesoporous silica material with different pore sizes or confinement sizes, respectively.

As a model reaction, a process was selected where the equilibrium constant can easily be obtained even though the molecules are located inside the porous host and which is already fully understood. Therefore, the excimer formation of pyrene molecules was chosen, that is, the complexation of an excited pyrene with a second pyrene molecule. The underlying excimer-monomer equilibrium has been extensively studied, ${ }^{18}$ and due to the very different fluorescence signals of the monomer and the excimer, experimental investigation of this equilibrium even inside a porous host is very easy, as several reports have already demonstrated. ${ }^{19,20}$ In addition, the pyrene-excimer system offers some other, well-known possibilities. For instance, pyrene can be used to probe the environment in a variety of systems, as in micelles, ${ }^{21,22}$ or pyrene was used to study the surface properties of inorganic oxides when adsorbed to these surfaces. ${ }^{23-25}$

Different from the previous reports, the objective of this paper is to study directly the influence of a spatial restriction on 


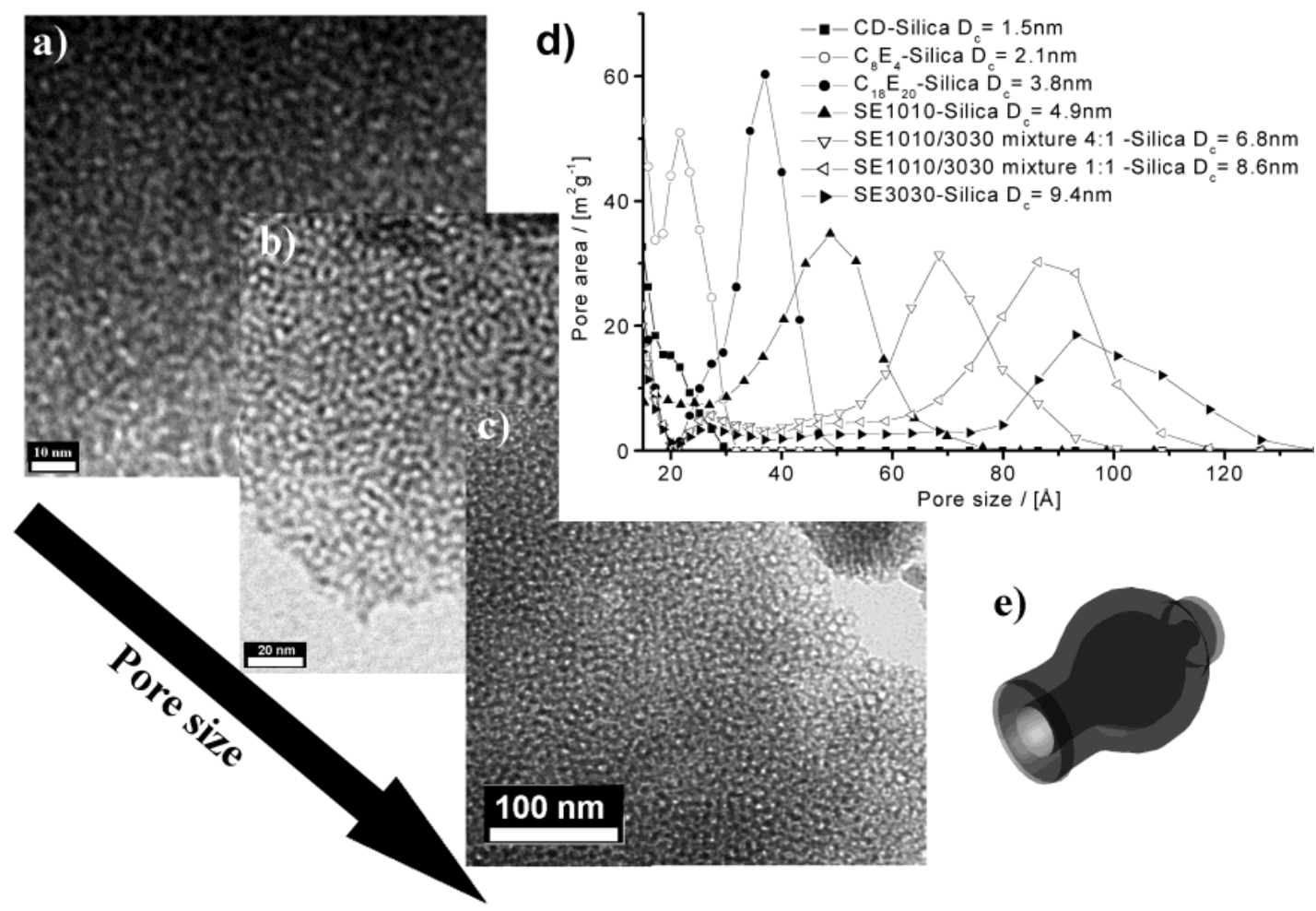

Figure 1. Introduction of the nanochemical reactors used in this study. Images a to c show TEM images of mesoporous silica materials with increasing pore size. Image a represents a cyclodextrin-based silica with $D_{\mathrm{c}}=1.5 \mathrm{~nm},{ }^{26}$ image b shows a Brij-based silica with $D_{\mathrm{c}}=3.8 \mathrm{~nm},{ }^{17}$ and image c is a SE-based silica with $D_{\mathrm{c}}=4.5 \mathrm{~nm} \cdot{ }^{17}$ Part d shows pore-size distribution functions derived by a density functional theory evaluation ${ }^{38}$ of sorption data. It is seen how the materials used in this study cover confinement sizes from 1.5 to $10 \mathrm{~nm}$. Image e illustrates a suitable model for the morphology of the mesopores.

chemical equilibria by statistical effects. Unlike previous studies, the current approach enables us to freely vary the size (and volume) of the spatial restriction in the range $1-10 \mathrm{~nm}$ without significant wall-substrate interactions being involved. For pyrene, in particular, it is qualitatively known that a spatial restriction influences the excimer formation, but quantitative statements were-due to the missing possibility of free reactor size variation-not possible. In addition, it is attempted to separate the influences of the spatial restriction from the influence of the dye-surface interactions by continuous variation of the wall polarity and the solvent character.

\section{Results and Discussion}

For the synthesis of the ordered mesoporous materials, we used the so-called nanocasting process where different template phases are transferred to the pores in a 1:1 imprinting process. ${ }^{11,17}$ Depending on the templates, different pore sizes can be achieved. Cyclodextrins (CDs) lead to pores between 1.5 and $2 \mathrm{~nm},{ }^{26}$ nonionic surfactants $\left(\mathrm{C}_{x} \mathrm{E}_{y}\right)$ or mixtures of them lead to pores between 2 and $4 \mathrm{~nm}$, and poly(styrene)-poly(ethyleneoxide) block copolymers (SE) result in pore diameters between 4.5 and $10 \mathrm{~nm}$ (see Figure 1). The materials were carefully analyzed by transmission electron microscopy (TEM), nitrogen sorption, and small-angle X-ray scattering (SAXS), the presentation of which is omitted here. For all template cases, the so-called "worm-type" pore morphology was found. These pores might be described best as an arrangement of spherical or prolate pores (the nanoconfinements) which are interconnected by smaller bottlenecks, thus forming a highly curved, bicontinuous pore system. The materials can be obtained as monolithic materials or in the form of thin films (see elsewhere). ${ }^{27}$ The macroscopic dimensions (cm-range) of the porous silica monoliths ensure that effects by adsorption on the external surface of the material are negligible. A certain variety of the surface properties of the mesoporous silica materials was obtained by calcination of samples performed at different temperatures ranging from $500{ }^{\circ} \mathrm{C}\left(T_{1}\right)$ to $525{ }^{\circ} \mathrm{C}\left(T_{2}\right)$, to 550 ${ }^{\circ} \mathrm{C}\left(T_{3}\right) .{ }^{29} \mathrm{Si}$ NMR measurements indicate that the pore walls of the silica materials calcined at lower temperatures possess a significant amount of silanol $(\mathrm{Si}-\mathrm{OH})$ groups and are therefore relatively polar, whereas the amount significantly decreases for $550{ }^{\circ} \mathrm{C}$.

The silica monoliths were freed from oxygen (a quencher) under high vacuum and heating with an air gun. The samples were contacted with degassed pyrene solutions using Schlenck techniques. After $2 \mathrm{~h}$, equilibration is assumed to have occurred, all pores are filled, the remaining solution is removed with a syringe, and the silica is washed with pure and oxygen free solvent to remove surface-adsorbed pyrene. The monolith pieces are placed in a closed cuvette for measurements.

The concentration dependence of the single pyrene to excimer equilibrium is examined by recording the fluorescence ratio $I_{\text {Exc }} /$ $I_{\mathrm{III}}$ (determined by the intensities of the excimer species at its maximum $\lambda=466 \mathrm{~nm}$ and the pyrene signal at $\lambda=392 \mathrm{~nm}$ ). In accordance with other contributions, the spectra were normalized to peak III. ${ }^{18}$ Another important parameter is the ratio $I_{\mathrm{III}} / I_{\mathrm{I}}$, which is an indicator for the polarity of the nanoenvironment of pyrene molecules. The silica pores are getting more hydrophobic with increasing calcination temperature $\left(I_{\mathrm{III} / \mathrm{I}}\left(T_{1}\right)=1.06 \rightarrow I_{\mathrm{III} / \mathrm{I}}\left(T_{3}\right)=1.6\right.$, which is more polar than heptane as the solvent $\left(I_{I I I / I}=2.9\right)$ and of the order of the polar solvent butanol). The effect of different solvents is mainly that the interactions of pyrene molecules with the silica wall can be triggered, as described later. To choose a solvent of 
a)

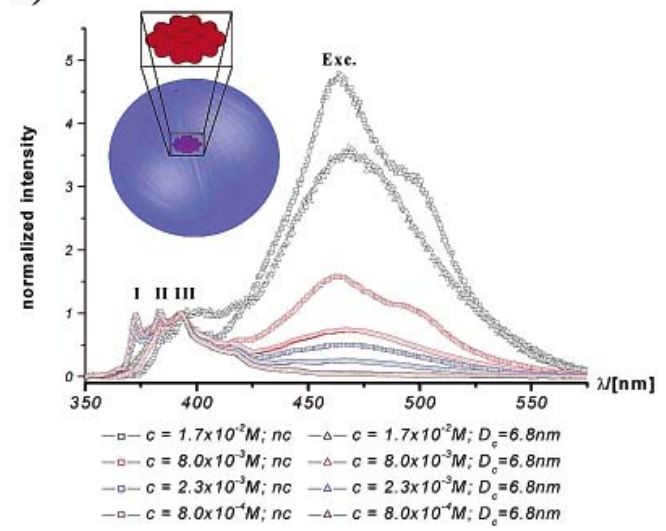

b)

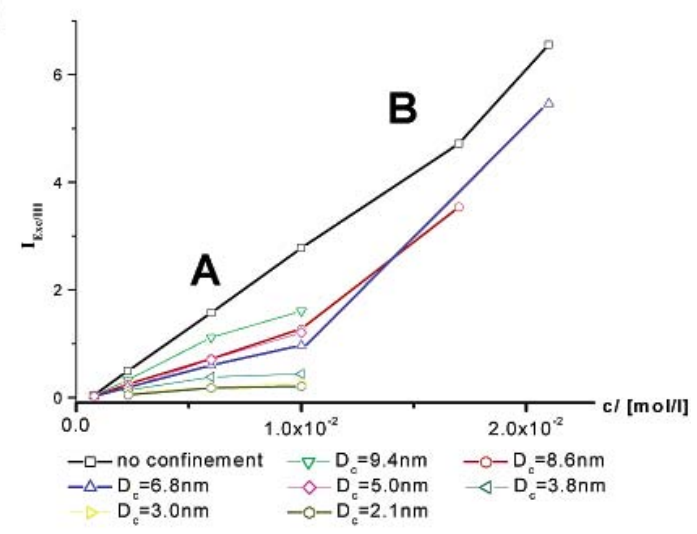

Figure 2. Concentration dependence of the formation of excimers. The confined state is compared to the unconfined state denoted n.c. Part a shows the emission spectra. The nomenclature of the emission signals (I, II, and III belonging to the pyrene monomer, and exc to the excimer) is given. The graphical inset in part a demonstrates the actual restriction a pyrene molecule experiences in a $7 \mathrm{~nm}$ pore. Part $\mathrm{b}$ shows directly the concentration dependence of the equilibrium given by $I_{\text {exc/III }}$ for different poresizes.

similar polarity to that of the silica walls leads to a situation where the adsorption and thus immobilization of pyrene at the pore walls can be neglected. The pyrene molecules are preferentially located in the pore volume, which means that the found effects can be attributed to the pure spatial restriction.

Figure $2 \mathrm{a}$ shows the difference between the fluorescence spectra of pyrene solutions ( $\mathrm{nc} \approx$ no confinement) with different concentrations and under confinement conditions $\left(D_{\mathrm{c}} \approx\right.$ diameter of the confinement $=$ pore size $)$. Note that the additional peak at longer wavelengths is due to the applied very high pyrene concentrations, which are, however, needed for the pore experiments. The spatial restriction on a single pyrene due to an $\approx 7 \mathrm{~nm}$ pore is illustrated by the simple geometrical comparison shown in the inset of Figure 2a. The pore size is comparable to that of a pyrene molecule but stills prevents enough space for excimer formation. Silica mesopores in the mentioned size regime should be able to influence molecular processes. The fluorescence signal corresponding to the excimer species is higher for all unconfined (ordinary) solutions than it is in the $6.8 \mathrm{~nm}$ pores. The spatial restriction suppresses the formation of excimers, and the equilibrium is shifted to single pyrene molecules.

Figure $2 \mathrm{~b}$ shows the concentration dependence of $I_{\mathrm{Exc}} / I_{\mathrm{III}}$ for different pore sizes. The excimer formation scales linearly ( $I_{\mathrm{Exc}} d$ $I_{\mathrm{III}} \propto c$ ) for pyrene in free solution, as expected from previous studies in the literature. In pores, pyrene exhibits a completely different and more complex behavior. The amount of excimer formation indeed depends on pore size: Qualitatively, the smaller the pores, the less excimer is formed, but no linear correlation to the concentration, as in the unconfined state, is found. It is also obvious from the given data that the excimer formation is more and more suppressed the smaller the pore size gets. In very small pores $\left(D_{\mathrm{c}} \approx 2 \mathrm{~nm}\right)$, the space and the separation in subvolumes are so strong that the formation of excimers is nearly completely hindered.

The data can be interpreted and understood in the following way. The pore system divides the macroscopic volume into nanometer-sized subvolumes. A formation of excimers which requires at least two pyrene molecules per pore is strongly hindered due to the separation of pyrene molecules to different confinements. This can be overcome either by increasing the concentration to a point where every pore contains already two molecules or by spectral molecular diffusion through the pore system toward states with two pyrene molecules. In very small pores $\left(D_{\mathrm{c}} \approx 2 \mathrm{~nm}\right)$, the space and the separation into subvolumes is so strong that this is even not possible for high concentrations, as the steadily low values for $I_{\text {Exc/III }}$ indicate. It should be noted again that nothing has been changed (concentration, temperature, etc.) except the division of the volume of measurement into nanometer-sized subcompartments.

The dependence of the excimer formation on the pore size is presented for three concentrations $\left(c_{1 / 2 / 3}\right.$, see Figure $\left.3 a\right)$ and compared to the case of unconfined solutions (continuous lines). The excimer formation stays below the values for the unconfined solutions in all cases. The formation of excimers is suppressed by $92 \%$ in small pores $(2 \mathrm{~nm})$ and by $44 \%$ in a $9.4 \mathrm{~nm}$ pore for $c_{1}$. For $c_{3}$ the suppression of excimer formation ranges from $88 \%$ to $31 \%$. In the very small pores, for all concentrations, comparably low values for $I_{\text {Exc/III }}$ are found. Here, due to the low signal, it cannot be excluded that also other factors than the fluorescence of embedded excimers contribute to the spectral response. There is just a small increase in $I_{\text {Exc/III }}$ when the pore size initially increases, although the volume of a confinement scales all the time scales with $D_{\mathrm{c}}{ }^{3}$. Then, eventually, a strong increase in $I_{\text {Exc/III }}$ for pore sizes near 4-5 nm can be seen (for $c_{1}$ ). For even larger pores, a plateau or an again slow rise of the excimer formation seems to be given. This transition in the excimer formation rate is very surprising and seems to be a unique feature due to confinement effects.

To understand this in a quantitative fashion, one might calculate the actual number of pyrene molecules per pore for the different concentrations, assuming spherical pore shape (for simplicity) and that the pyrene concentration is not changed due to the infiltration process. If done so, the excimer formation rate $I_{\mathrm{Exc}} I_{\mathrm{III}}$ can be plotted over the average number of pyrene molecules per pore for all pore sizes (Figure $3 b$ ).

At first, it is seen that the described experiments indeed describe the relevant range for "single molecule" experiments; that is, the average number density is between 0.01 and 2 pyrene molecules per pore. Second, one can easily see that despite the very different pore volumes (covering about 2 orders of magnitude) and potential differences in pore geometry, the data, nevertheless, seem scattered around one master curve. The remaining differences are potentially due to surface effects, which will be discussed below in more detail. Some type of increased excimer formation rate is observed when the average number of pyrene molecules per pore approaches one. It is interesting to compare this curve with the probability of having two pyrene molecules in one pore, which can be calculated according to simple Poisson statistics (see Figure 3b). Obviously, 

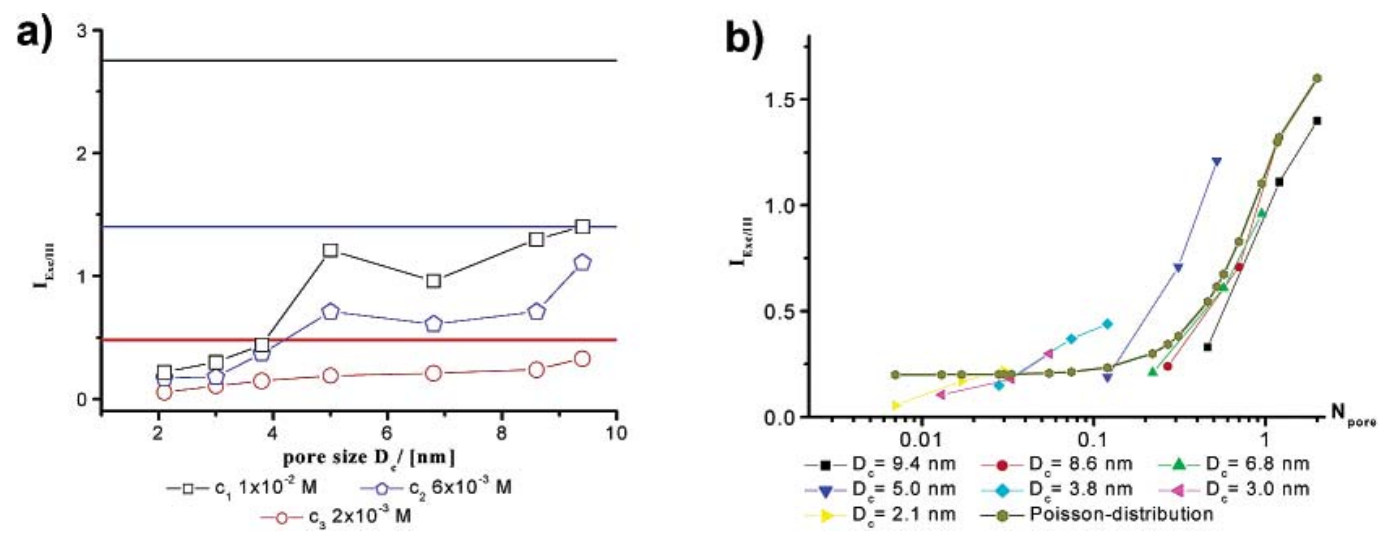

Figure 3. Graph a shows the dependence of the excimer formation ration expressed via $I_{\text {exc/III }}$ against the pore diameter ranging from 2 to $10 \mathrm{~nm}$. Three concentrations were investigated. The levels of $I_{\text {exc/III }}$ for the unconfined solutions of pyrene in butanol are shown by the straight and constant lines. The number of pyrene molecules per pore $N_{\text {pore }}$ is set in relation to $I_{\text {exc/III }}$ in graph b. For this graph the data from Figure $3 \mathrm{a}$ (pore size dependence) and the data from Figure 2 (concentration dependence) were taken into account.

the excimer formation rate goes with this probability; that is, excimer formation simply relies on the presence of a second pyrene molecule. However, if one additionally correlates the single data points in Figure $3 b$ to the pore sizes of the used materials (color-coded), it seems that some material specific effects are also present. For high number densities, the points seem to fit to the Poisson distribution very well independently of the used material. For low number densities of pyrene (respectively, materials with small pores), there is just a qualitative agreement. Unfortunately, we were not able to investigate higher concentrations in small pores due to limitations in the experimental/analytical setup. These data points would be necessary to judge if really all data points lie on one Poisson master curve. There is a possibility that for different materials separate Poisson curves exist due to differences in the free paths for pyrene, which can be different from material to material. Unexpectedly, for very low pyrene numbers per pore, a fluorescence in this spectral range is still detectable, which can be either due to other sources of fluorescence or due to spectral hopping diffusion of the excited state toward the rare pores with two pyrenes closely tight to each other, that is, a case selected-by-confinement, which is rare in the nonconfined solution but occurs with a higher probability in small pore systems (if there are two in a single pore, they are automatically close to each other).

To clarify the question if the observed effects possess a static contribution (i.e. the formation of so-called ground-state dimers due to confinement, as they are found in the cavities of cyclodextrins), ${ }^{28}$ further measurements are necessary.

Wavelength-dependent (emission and excitation) spectra were recorded, where for the $\mathrm{CD}$-entrapped pyrene units typical dependencies were described.$^{28}$ For pyrene in supercritical $\mathrm{CO}_{2}$, on the other hand, the normalized emission wavelengthdependent excitation spectra were identical for monomer and excimer, indicating no contribution of ground-state dimers. ${ }^{28}$

We investigated the influence of the three excitation wavelengths 325,333 , and $350 \mathrm{~nm}$ and compared the results directly to the data presented in ref 28. It was found (data not shown) that the normalized intensity of the excimer only slightly changes with changing excitation wavelength, indicating that a minority fraction of ground-state dimers is indeed present. This is supported by the normalized excitation spectra recorded for $\lambda_{\mathrm{em}^{-}}$ $(\mathrm{exc})=460 \mathrm{~nm}$ and $\lambda_{\mathrm{em}}(\mathrm{pyr})=380 \mathrm{~nm}$, which slightly differ from each other as well. However, the differences are much smaller than those for pyrene/cyclodextrins complexes. Therefore, static contributions to the excimer formation are present and might explain the overexpression of excimer fluorescence at very low relative dye numbers. In any case, the overall concentration dependence of excimer formation is by far too high to be explained by a static distribution model and indicates that the presented mesoporous system does not fully restrict the movement of excited pyrene molecules to neighboring pores (a simple back of the envelope calculation on the basis of the fluorescence lifetime presented below gives an average travel distance of $25 \mathrm{~nm}$ ). It is interesting to note in this context that the "transition" in Figure 3 b occurs at a relative number density of 1 pyrene molecule per pore.

An estimation of the importance of surface effects in such experiments can be obtained by variation of the polarity of both the silica wall material and the solvent. The polarity of the walls can be changed by calcining the silica materials at different temperatures, by which the content of surface silanol groups is varied. We calcined our materials at different temperatures $T$ $=450{ }^{\circ} \mathrm{C}, 500{ }^{\circ} \mathrm{C}$ (denoted $\left.T_{1}\right), 525{ }^{\circ} \mathrm{C}\left(\mathrm{T}_{2}\right), 550{ }^{\circ} \mathrm{C}\left(T_{3}\right)$, and $800{ }^{\circ} \mathrm{C}$ under otherwise constant conditions (oxygen atmosphere). The differently calcined materials were characterized with TEM, nitrogen sorption, IR, Raman, and ${ }^{29} \mathrm{Si}-\mathrm{MAS}$ NMR. Materials at lower calcination temperatures $\left(\leq 450{ }^{\circ} \mathrm{C}\right)$ were prepared, but due to some carbon residues leading to colors ranging from orange to deep black, these compounds were not further considered. ${ }^{27}$ It was also found that the internal surface area, pore volume, and pore morphology practically do not change between 450 and $550{ }^{\circ} \mathrm{C}$, whereas the materials collapsed for temperatures higher than $800{ }^{\circ} \mathrm{C}$. By ${ }^{29} \mathrm{Si}-\mathrm{MAS} \mathrm{NMR}$, it was found that the content of the fully condensed (silanol-free) $q^{4}$ groups in relation to $q^{3+2}$ groups evolves from $q^{4} / q^{3+2}=$ 1.625 at $T=500{ }^{\circ} \mathrm{C}$ to $q^{4} / q^{3}=2.5$ at $T=550{ }^{\circ} \mathrm{C}$, while $q^{1}$ units are practically absent. These findings were also supported by IR (narrowing of the band at $\approx 3750 \mathrm{~cm}^{-1}$ for higher temperatures) and Raman measurements (change of relative intensity of the bands at 784 and $1150 \mathrm{~cm}^{-1}$ ). This is in good agreement with the literature..$^{29-31}$

Besides the $\mathrm{OH}$ content of the walls, also the solvent was varied. The resulting fluorescence data are summarized in Figure 4. The formation of excimers is practically suppressed when either a solvent with low polarity (see Figure 4a for heptane) or an unpolar silica is chosen. For instance, the excimer formation rate stays low for heptane as a solvent, independently from the pore size, unlike the previous findings in butanol. In both cases for low polarity, it is reasonable to assume that the pyrene molecules adsorb to the silica walls under these conditions. Indeed, pyrene shows high retention on silica TLC 

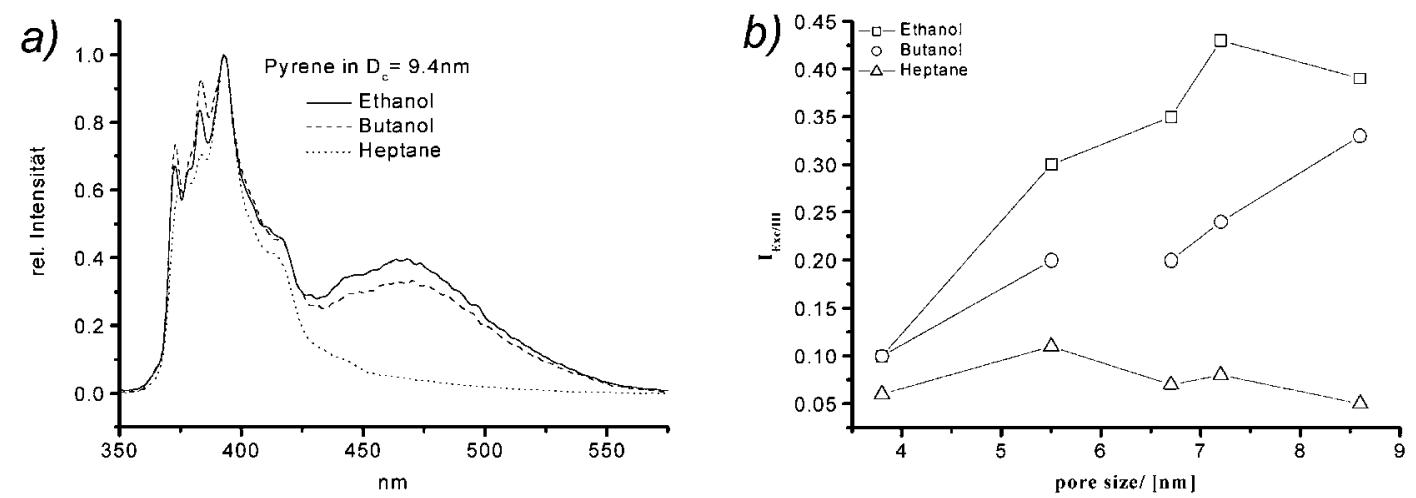

Figure 4. (a) Fluorescence spectra for pyrene in different solvents for $c=6 \times 10^{-3} \mathrm{M}$ in a $9.4 \mathrm{~nm}$ confinement and (b) the direct pore size dependence.

a)

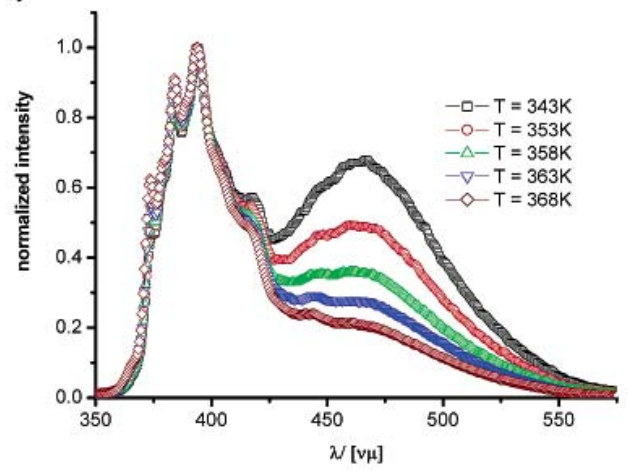

b)

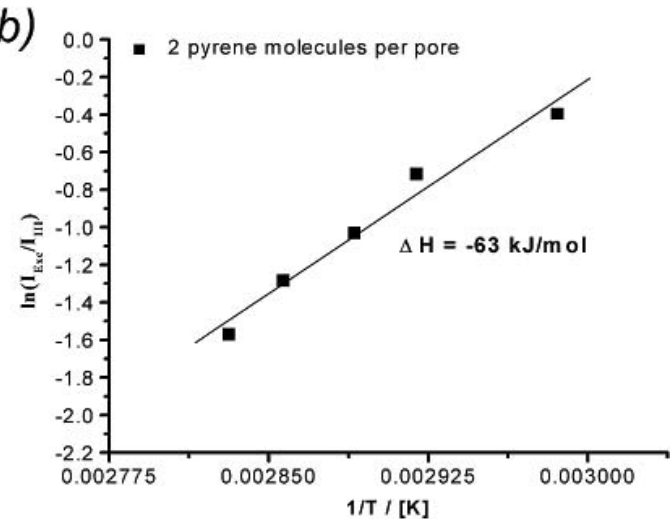

Figure 5. Temperature dependence of the pyrene-excimer equilibrium (a) and a plot of $\ln \left(I_{\mathrm{exc}} I_{\mathrm{III}}\right)$ against $1 / T$ to determine the enthalpy (b) for the cases when two pyrene molecules are located in a mesopore.

plates when hexane is used, while it shows practically no retention for alcoholic solvents and hydrated silica; the adsorption of pyrene was used as a characteristic feature in many other studies. $^{23,32,33}$ It is simply that, in these cases, the adsorbed pyrenes are highly reduced in mobility and cannot approach a second pyrene for excimer formation. Changing from butanol to the more polar ethanol, only a minor increase of excimer formation is found. These experiments give a belated reason for our choice of solvent (butanol) and wall material (calcined at $500{ }^{\circ} \mathrm{C}$ ) to analyze confinement effects, as unperturbed as possible by surface adsorption effects.

Having found appropriate conditions for successful compartmentalization where the pores simply separate pyrene molecules from each other, it is interesting to analyze the thermodynamic parameters of this process, such as the free enthalpy of excimer formation $\Delta G_{\text {exc }}$. Thermodynamic functions such as free enthalpy, enthalpy, or most likely entropy are functions of the partition function $Z$ of the corresponding system. A change in equilibrium constant, and in $\Delta G_{\mathrm{exc}}, \Delta H_{\mathrm{exc}}$, or $\Delta S_{\mathrm{exc}}$, respectively, by a chemical confinement is therefore related to a change in the statistical situation which will affect the partition function; or in other words, the number of realization possibilities for both monomer and excimers is changing by the confinement.

By investigating the temperature dependence of the excimer equilibrium, thermodynamic functions were determined for confined states and compared to data for the unconfined state of pyrene in butanol. The methods were directly adapted from the literature. ${ }^{34-36}$ In particular, we used the approach by Stevens and Ban, which allows us to determine the enthalpy and entropy from the temperature dependence of the fluorescence spectra. ${ }^{34}$ First, the enthalpy $\Delta H_{\text {exc }}$ was determined by a plot of $\ln \left(I_{\text {exc/III }}\right)$ against $T^{-1}$ (see ref 37 ), and then $\Delta S_{\text {exc }}$ was determined according to the Stevens-Ban method expressed as

$$
\ln \left(\frac{I_{\text {exc }}}{I_{\text {III }}}\right)=\ln \left(c_{\text {pyrene }} a\right)+\frac{\Delta S_{\text {exc }}}{R}-\frac{\Delta H_{\text {exc }}}{R T}
$$

The parameter $a$ in eq 1 is the slope of the curve when $I_{\text {exc }}$ is plotted against $I_{\mathrm{III}}$ for the different temperatures (plots not shown). The temperature dependence of excimer formation on the basis of which $\Delta H_{\mathrm{exc}}$ and $\Delta S_{\mathrm{exc}}$ were determined is exemplary shown in Figure 5 for the situation where, on average, two molecules of pyrene are housed in one $5 \mathrm{~nm}$ pore.

Similar to the situation in free solution, the concentration of excimer decreases when the temperature rises. The determined enthalpy for excimer formation inside $5 \mathrm{~nm}$ pores is $-63 \mathrm{~kJ} /$ $\mathrm{mol}$, about $30 \mathrm{~kJ} / \mathrm{mol}$ lower than that for the unconfined state. Obviously, the excimer is stabilized by the confinement, as indicated by the increased heat of formation. This can be either a bare geometric effect or adsorption of the excimer onto the silica wall, generating heat and increasing its stability. The slight difference in enthalpy for the "free" solution in comparison to values reported in the literature ${ }^{34-36}$ can be understood by the fact that we just determined the lower end of the possible temperature range, as compared to these reports.

Calculating the entropy for this process from the intercept in Figure 5, an apparent change from $\Delta S_{\mathrm{exc}}=-37 \mathrm{~J} \mathrm{~K}^{-1} \mathrm{~mol}^{-1}$ for the normal solution to $\Delta S_{\mathrm{exc}}=-205 \mathrm{~J} \mathrm{~K}^{-1} \mathrm{~mol}^{-1}$ for a 5.0 $\mathrm{nm}$ confinement is found. Taking these values into account would result in a positive free enthalpy of excimer formation, that is, no formation of excimers at all. If one would even select a state with less than 1 pyrene molecule per pore (before the 

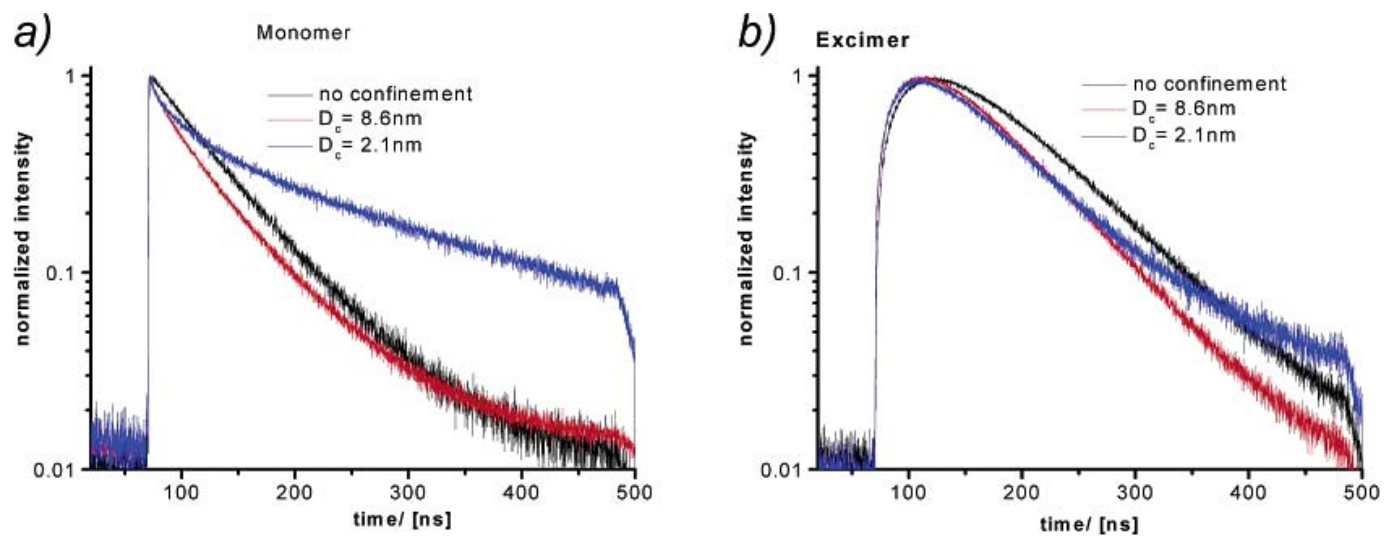

Figure 6. Lifetime measurements of the monomer species (pyrene) (a) and the excimer species (b).

transition in Figure 3b; data not shown), the enthalpy and entropy become apparently even more unfavorable for excimer formation. However, the "strange" values obtained for the thermodynamic functions indicate that an evaluation of the data within the traditional formalism of the canonical ensemble is not suitable (as practically proven by the formally positive free energy of excimer formation). It seems much more the case that the calculation of the entropy of excimer formation has to consider not the complete phase space but just the adoptable coordinates, which obviously changes due to confinement. The statistical influence of confinement on the thermodynamic functions can potentially be treated as the introduction of a microcanonic ensemble in the Gibbs formalism. As our data material is not sufficient to model the data in a quantitative fashion, we restrict ourselves to the statement that the classical thermodynamic approach can obviously not be applied to the present case of porous media.

It is further interesting to determine other properties of this very special state of confined single pyrene molecules or excimers. In lifetime experiments, some other peculiarities become visible, as shown in Figure 6. For the fluorescence of pyrene monomers, it is seen that already nanofinement within $8.6 \mathrm{~nm}$ pores, which remains practically unseen in the formation of excimers, is seen in the fluorescence lifetime: the formerly strictly monoexponential curve is slightly broadened, that is, contains faster and slower contributions. This can be explained by an altered, heterogeneous dielectric environment. The pyrenes in the $2.1 \mathrm{~nm}$ pores show a completely different relaxation behavior: there is a fastened relaxation mode similar to the one in $8.6 \mathrm{~nm}$ pores, running into a homogeneous slow process with a lifetime of $t_{2}=19.4 \mathrm{~ns}$, an increase of the lifetime by a factor of 3. A potential explanation of this effect is the stabilization of the more polar excited state by the silica walls, as already discussed above. A similar effect is seen for the lifetime of the excimers (Figure 5b), which shows the typical profile of a reactive intermediate. Buildup and primary decay are a little faster for all the excimers within the silica pores, while the excimer confined in $2.1 \mathrm{~nm}$ silica shows a pronounced second relaxation mode with an extended fluorescence lifetime corresponding to the one of the monomer. Therefore, also the excimers inside a $2.1 \mathrm{~nm}$ spatial confinement are more stable and have to decay according to a different mechanism. For the measurements in the $8.6 \mathrm{~nm}$ pores, the lifetimes of the excited monomers and the excimers are practically identical to those of the unconfined state. A quantitative description of the data is very hard to perform due to the complex mechanism of excimer formation. However, it is interesting to discuss the lifetime measurements in the context of the microcanonic situation, as mentioned above. A higher lifetime of excited species under confinement indicates not necessarily an altered enthalpy, but can also be due to a difference in entropic decay paths (usually subsummarized as "solvent effects") which contribute to the lifetime of the excited state, too.

\section{Conclusion}

It was shown that excimer formation of pyrene in mesoporous silica under appropriate solvent and surface energy conditions is a model case to examine the influence of a spatial confinement on a bimolecular reaction, revealing a whole variety of peculiarities and effects.

Although the presented confinement conditions were not ideal despite a real adiabatic separation of single species, it could be shown that the thermodynamics of excimer formation are altered by the spatial confinement, which, in addition, depends on the pore size and pore loading.

A significant suppression of excimer formation was described, which, however, stayed unexpectedly high at average pyrene per pore concentrations, much higher than expected by simple Poisson statistics. This was speculatively explained by spectral hopping toward rare dimer states, as a (usually negligible) minority fraction of ground state dimers could be spectroscopically traced.

Fluorescence lifetime measurements of pyrene in $2.1 \mathrm{~nm}$ pores showed a stabilization and increased lifetime of both pyrene and excimer fluorescence. It is interesting to speculate if this stabilization is due to confinement effects (restricting entropic decay paths, solely) or due to simple enthalpic adsorption of the excited species on the nearby silica walls. These two effects are, however, experimentally not easily separated, as the resulting change of the fluorescence spectrum remains unresolved.

The higher stability of excimers is also expressed by a more exothermic heat of excimer formation, as determined from temperature-dependent measurements. Here, $\Delta H_{\mathrm{exc}}$ inside 4.5 $\mathrm{nm}$ pores was found to be $-63 \mathrm{~kJ} / \mathrm{mol}$, and therefore, about 30 $\mathrm{kJ} / \mathrm{mol}$ lower than that in the unconfined state. The difference of about $12 k T$ would compare favorably both with typical heat of adsorptions and with the expected entropic confinement effects, for which the calculation of enthalpy has not been corrected.

Problems with the Stevens-Ban method to determine enthalpy and entropy of excimer formation indicate that this classical description may not be applicable to systems with a restricted phase space, that is, in a confinement which has the same spatial extension as that of the ongoing process. Further 
work has to be attributed to evaluating different microcanonical corrections to adjust to the confined situations, which, however, rely on an extended set of measurements.

\section{Experimental Part}

Materials. All precursors were obtained from Aldrich in the highest available purity grade and used without further purification. The production of mesoporous silica materials as well as pyrene loading is described in the cited references or mentioned in the text. It is denoted that the silica materials calcined at low temperatures might still contain organic impurities not visible to the eye but UV/vis active. This impurities can significantly influence the obtained fluorescence spectra. Therefore, these materials were treated with concentrated $\mathrm{H}_{2} \mathrm{O}_{2}$ after calcination until the UV/vis spectra of the materials were identical to those of pure silica glass. The structural integrity of the materials toward this process was checked by nitrogen sorption and TEM.

Measurements. Transmission electron microscopy (TEM) images were acquired on a Zeiss EM $912 \Omega$ at an acceleration voltage of $120 \mathrm{kV}$. Samples were ground in a ball mill and taken up in acetone. One droplet of the suspension was applied to a 400 mesh carbon-coated copper grid and left to dry in air. Nitrogen sorption data were obtained with a Micromeritics Tristar instrument. Small-angle X-ray scattering (SAXS) curves were recorded by means of a Kratky camera and also a rotating anode with pinhole collimation. A Nonius rotating anode device $(P=4 \mathrm{~kW}, \mathrm{Cu} \mathrm{K \alpha})$ and an image-plate detector system were used. With the image plates placed at a distance of $40 \mathrm{~cm}$ from the sample, a scattering vector range from $s=0.05$ to $1.6 \mathrm{~nm}^{-1}$ $(s=2 / \lambda \sin \theta)$ was available. The samples were irradiated for $18 \mathrm{~h}$ to reduce the noise level and to obtain a sufficiently high scattering intensity. The 2D isotropic diffraction patterns were transformed into a 1D radial average of the scattering intensity. ${ }^{29} \mathrm{Si}$-MAS NMR spectra were acquired on a Bruker Avance DPX 400 spectrometer. IR spectra were recorded on a Biorad FTS 600 FTIR spectrometer. Ungrounded material fragments were used for the analysis. We used a Perkin-Elmer luminescence spectrometer LS 50B for the fluorescence measurements. The lifetime measurements were performed on a FLS 920 spectrometer from Edinburgh Instruments. Diffuse reflection spectroscopy measurements in the UV/vis/near-IR region were carried out on a Jasco VERY-570 spectrophotometer equipped with an integrating sphere. Spectra were recorded at room temperature from 200 to $900 \mathrm{~nm}$ with a scanning speed of 100 $\mathrm{nm} / \mathrm{min}$ using $\mathrm{MgCO}_{3}$ as a reference. Raman spectra were obtained on a confocal Raman microscope from Witec. The Raman spectrometer is equipped with a $15 \mathrm{~mW}$ laser source $(\lambda$ $=532 \mathrm{~nm}$ ). The carbon sample was put on a microscope slide and covered with a glass cover. Direct scattering is detected using a CCD detector system.

Acknowledgment. The authors would like to thank Prof. Löhmannsröben and Dr. M. Kumke (University of Potsdam) for giving us the opportunity to perform the lifetime measurements of the excited species. The Max-Planck society is acknowledged for financial support.

\section{References and Notes}

(1) Tersoff, J. Nature 2001, 412, 135.

(2) Ball, P. Nature 2000, 408, 904.

(3) Sohn, L. L. Nature 1998, 394, 131.

(4) Ozin, G. A. Adv. Mater. 1992, 4, 612.

(5) Whitesides, G. Technol. Rev. 1998, 101, 84.

(6) Ozin, G. A.; Chomski, E.; Khushalani, D.; Maclachlan, M. J. Curr. Opin. Colloid Interface Sci. 1998, 3, 181.

(7) Hue, Q. S.; Margolese, D. I.; Ciesla, U.; Feng, P. Y.; Gier, T. E.; Sieger, P.; Leon, R.; Petroff, P. M.; Schüth, F.; Stucky, G. D. Nature 1994, 368,317 . 131.

(8) Ciesla, U; Schüth, F. Microporous Mesoporous Mater. 1999, 27,

(9) Schüth, F. Curr. Opin. Colloid Interface Sci. 1998, 3, 174.

(10) Polarz, S.; Antonietti, M. Chem. Commun. 2002, Advance Article, 2593.

(11) Göltner, C. G.; Henke, S.; Weissenberger, M. C.; Antonietti, M. Angew. Chem. 1998, 110, 633.

(12) Melosh, N. A.; Lipic, P.; Bates, F. S.; Wudl, F.; Stucky, G. D.; Fredrickson, G. H ; Chmelka, B. F. Macromolecules 1999, 32, 4332.

(13) Feng, P.; Bu, X.; Stucky, G. D.; Pine, D. J. J. Am. Chem. Soc. 2000, 122, 994.

(14) Junges, U.; Jacobs, W.; Voigtmartin, I.; Krutzsch, B.; Schuth, F. J. Chem. Soc., Chem. Commun. 1995, 2283.

(15) Beck, J. S.; Vartuli, J. C.; Roth, W. J.; Leonowicz, M. E.; Kresge, C. T.; Schmitt, K. D.; Chu, C. T.; Olson, D. H.; Sheppard, E. W.; McCullen,

S. B.; Higgins, J. B.; Schlenker, J. L. J. Am. Chem. Soc. 1992, 114, 10834. (16) Kresge, C. T.; Leonowicz, M.; Roth, W. J.; Vartuli, J. C.; Beck, J. S. Nature 1992, 359, 710 .

(17) Smarsly, B.; Polarz, S.; Antonietti, M. J. Phys. Chem. B 2001, 105, 10473.

(18) Förster, T. Angew. Chem., Int. Ed. 1969, 8, 333.

(19) Keeling-Tucker, T.; Brennan, J. D. Chem. Mater. 2001, 13, 3331.

(20) Dunn, B.; Zink, J. I. Chem. Mater. 1997, 9, 2280.

(21) Lianos, P.; Zana, R. J. Phys. Chem. 1980, 84, 3339.

(22) Kwon, G.; Naito, M.; Yokoyama, M.; Okano, T.; Sakurai, Y.; Kataoka, K. Langmuir 1993, 9, 945.

(23) Mao, Y; Thomas, J. K. Langmuir 1992, 8, 2501.

(24) Rutan, S. C.; Harris, J. M. J. Chromatogr., A 1993, 656, 197.

(25) Zilberstein, J.; Bromberg, A.; Berkovic, G. J. Photochem. Photobiol., A Chem. 1994, 77, 69.

(26) Polarz, S.; Smarsly, B.; Bronstein, L.; Antonietti, M. Angew. Chem., Int. Ed. 2001, 40, 4417.

(27) Mastai, Y.; Polarz, S.; Antonietti, M. Adv. Funct. Mater. 2002, $12,197$.

(28) Zagrobelny, J.; Betts, T. A.; Bright, F. V. J. Am. Chem. Soc. 1992, 114,5249 .

(29) Oepen, S. B.; Gunther, H. Magn. Reson. Imaging 1996, 14, 993. (30) Holtz, F.; Beny, J. M.; Mysen, B. O.; Pichavant, M. Chem. Geol. 1996, 128,25 .

(31) Okamoto, M.; Nobuhara, K.; Jinno, K. J. Chromatogr. 1991, 556, 407.

(32) Wellner, E.; Ottolenghi, M.; Avnir, D. Langmuir 1986, 2, 616

(33) Lochmüller, C. H.; Colborn, A. S.; Hunnicutt, M. L.; Harris, J. M. J. Am. Chem. Soc. 1984, 106, 4077.

(34) Stevens, B.; Ban, M. I. Trans. Faraday Soc. 1964, 60, 1515.

(35) Förster, T. Angew. Chem., Int. Ed. 1960, 72, 716.

(36) Förster, T.; Kasper, K. Z. Elektrochem. 1955, 59, 976

(37) Birks, J. B. Photophysics of Aromatic Molecules; Wiley-Interscience: London, 1970.

(38) Ravikovitch, P. I.; Haller, G. L.; Neimark, A. V. Adv. Colloid Interface Sci. 1998, 76-77, 203. 